\title{
Vernal keratoconjunctivitis in the black child and its response to therapy
}

\author{
E. DAHAN AND R. APPEL \\ From the Department of Ophthalmology, University of the Witwatersrand, Johannesburg, and \\ St John's Eye Unit, Baragwanath Hospital, Soweto, South Africa
}

SUMmARY A clinical study of vernal keratoconjunctivitis in black children in Southern Africa was conducted to report on the peculiar, predominantly limbal form of the disease, and to test its response to therapy. Pigmented and thickened limbal conjunctiva gave the disease its typical appearance. In severe cases these lesions were encroaching on to the cornea and threatened sight. Placebo had no effect on the course of the disease. Topical steroids and sodium cromoglycate were equally effective. A combination of steroids and SCG proved particularly effective in treating severe cases, indicating a possible synergistic effect of the 2 drugs.

Vernal keratoconjunctivitis (VKC) is a recurrent external ocular disease of unknown aetiology, often characterised by seasonal exacerbations. The patient, usually in the first or second decade of life, usually a male, complains of itching, grittiness, watering, and photophobia. Diagnostic signs include a thick, stringy, mucous discharge, a giant 'cobblestone' papillary reaction of the upper tarsal conjunctiva, papillary changes of the bulbar conjunctiva, especially at the upper limbus, and corneal pathology, ranging from superficial punctate keratitis (SPK) through ulceration and plaque formation.

Many patients have a personal or family history of atophy, and the disease has been characterised as a form of type I (immediate) hypersensitivity. ${ }^{1}$ Therapy has centred about the use of topical corticosteroids, ${ }^{2}$ but there have been many encouraging recent reports of the use of sodium cromoglycate (SCG) eye drops in VKC ${ }^{3-7}$ both to control the acute manifestations of the disease and to reduce the patients' dependence on the use of corticosteroids, which carry with them the attendant risks of infections and glaucoma.

Most patients studied, usually of Western European background, display the typical picture of VKC as described above. But some have a rather different, though equally striking picture. They show a circumcorneal, pigmented limbal thickening, sometimes encroaching on to the cornea, practically or totally to the exclusion of other findings (see Figs.

Correspondence to Dr E. Dahan, Department of Ophthalmology, Medical School, York Road, Park Town 2193, Johannesburg, South Africa.
$1,2,3)$. We have noted a high incidence of VKC in the black patients attending the clinics in Southern Africa, almost all with this limbal form of the disease. We have taken the opportunity to describe this peculiar form of the disease and its response to therapy.

\section{Material and methods}

Black patients screened in a general outpatient eye clinic who were found to have active VKC were referred to one of the authors. These patients had neither been seen before nor been treated for at least 6 months prior to our study. Patients with other external eye diseases were excluded. Patients (or the patient's family in the case of the younger ones) were questioned about the duration and variation with the seasons of their condition. A family history of VKC or allergies was elicited, as well as a personal history of asthma, eczema, hay fever, or rhinitis.

Patients were asked to rate their symptoms of itching, tearing, and photophobia as to severity, and were scored on a basis of 0 to 4 for each symptom $(0=$ not present,$\quad 1=$ mild, $2=$ moderate, $3=$ moderate/severe, $4=$ severe). On each visit the patients were examined by slit-lamp biomicroscopy, and by fluorescein staining when indicated, and each eye was rated on the same $0-4$ scale based on the severity of pathological changes observed in the tarsal, bulbar, limbal conjunctiva, and the cornea. Selected patients had conjunctival scrapings or conjunctival biopsies of involved areas taken. 
After their initial assessment patients were randomly given either placebo (normal saline), corticosteroid (dexamethasone $0 \cdot 1 \%$ ), or sodium cromoglycate $2 \%$ (Opticrom) eye drops to be used 4 times a day in both eyes, and were seen at 2-weekly intervals. If little or no response to therapy was noted after 4 weeks, the patients using placebo were switched to one of the active compounds, while patients using dexamethasone or SCG were given the other drug to be used concurrently. Follow-up periods ranged from 2 to 8 weeks. At the conclusion of the study, the scores for each symptom and sign, and the total subjective and objective scores, were averaged for each treatment regimen to determine their relative efficacy.

\section{Results}

A total of 80 patients were examined for the study. Of these, 40 had sufficient follow-up to be included in the therapeutic assessment.

\section{CLINICAL FEATURES}

The age of the patients ranged from $2 \frac{1}{2}$ years to 18 years with an average age of $71 / 2$ years. $80 \%$ were urban and $20 \%$ rural, but this fact is due to the proximity of St John's Eye Hospital to Soweto. $75 \%$ were male and $25 \%$ female.

The duration of the disease varied from a few months to 15 years but was mainly a function of the age of the patient. The average duration was 3 years, and the severity of the signs and symptoms correlated well with the duration of the disease (see Figs. 3, 4, 5, 6).

\section{SEASONAL VARIATION}

$35 \%$ of the patients reported worsening of symptoms during summer, which is a hot and rainy season. $15 \%$ reported worsening of symptoms during winter, which is a dry and cold season. $35 \%$ reported to suffer

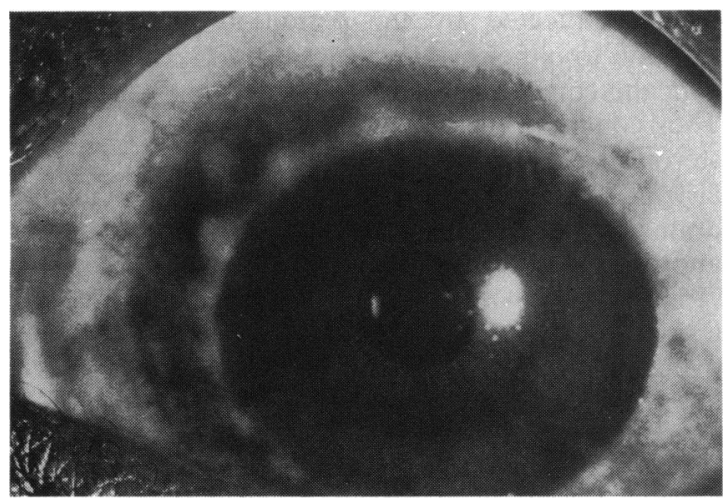

Fig. 1 7-year-old patient with VKC. Note the thickened and pigmented limbal conjunctiva.

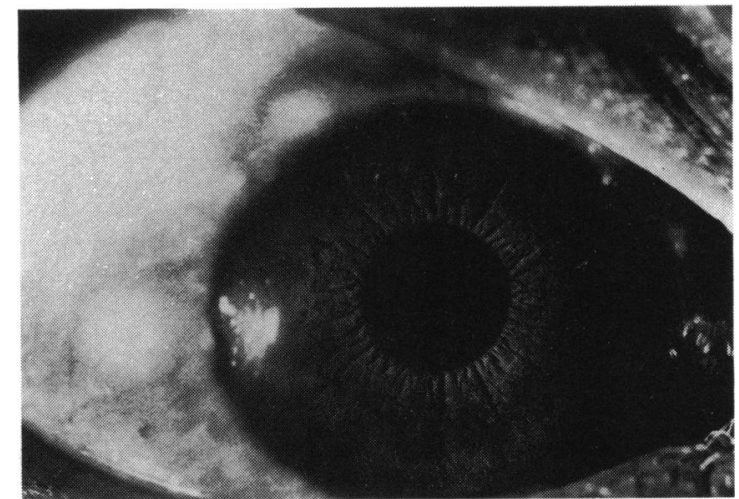

Fig. 2 8-year-old patient with VKC. Note the succulent appearance of the limbus.

equally throughout the year. $15 \%$ could not specify any seasonal variation. $25 \%$ had a positive family history of VKC. Only 5\% reported an associated hay fever rhinitis, but no other atopic conditions were found.

The symptoms included intense itchiness, tearing, and photophobia. A mucous discharge was occasionally reported, but the main reasons which brought the patient to the Eye Clinic were persistent itching of the eyes and the typical appearance of the pigmented and thickened pericornea (Figs. 1, 2, 3).

On biomicroscopy the bulbar conjunctiva appeared to be oedematous with variable engorgement of blood vessels. There was a typical distribution of pigment around the limbus corresponding to the interpalpebral fissures (Figs. 1, 2). The limbal conjunctiva was thickened, giving a succulent appearance (Fig. 2) and in severe cases encroaching on the cornea (Fig. 3).

The corneal involvement consisted mainly of superficial punctate keratopathy (Fig. 4). In our series no ulceration or plaque formation was noted.

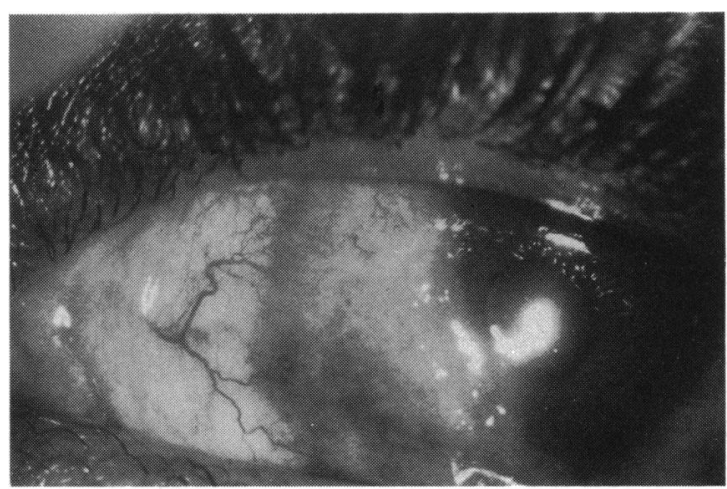

Fig. 3 14-year-old girl suffering from severe VKC. Note the encroaching of the limbal lesion on to the cornea, in which case the disease may threaten sight. 


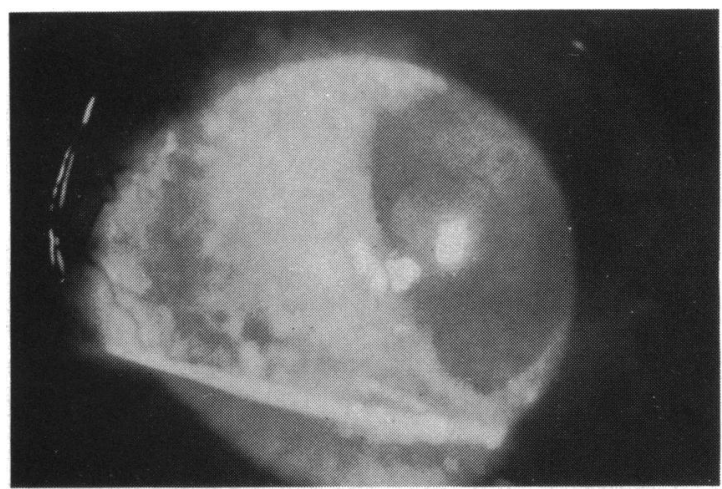

Fig. 4 Same patient as in Fig. 3, eye stained with fluorescein. Note the patchy staining of the limbal lesion and the superficial punctate keratopathy.

The palpebral conjunctiva showed mild papillary reaction, but the typical giant cobblestones appearance was practically never noted in the black children in our series (Fig. 5).

The giant papillae were seen only in Asiatic patients, who were excluded from our study.

Fluorescein staining of the lesions at the limbus and cornea reflected the activity of the disease (Fig. 4) and correlated well with the severity of the symptoms. The histology of biopsies at the limbus revealed: (1) an alternating hypertrophy and necrosis of the conjunctival epithelium; (2) a cellular infiltration in the stroma consisting mainly of eosinophils, monocytes, and plasma cells. Conjunctival scrapings showed conjunctival epithelial cells mixed with eosinophils. Therapeutic assessment was reasonably accurate in relation to the objective signs because of the direct visualisation of the pathology, which was mainly limbal, therefore easily accessible; whereas the authors had to rely on the report of the patients or their guardians for the scoring of the subjective symptoms.

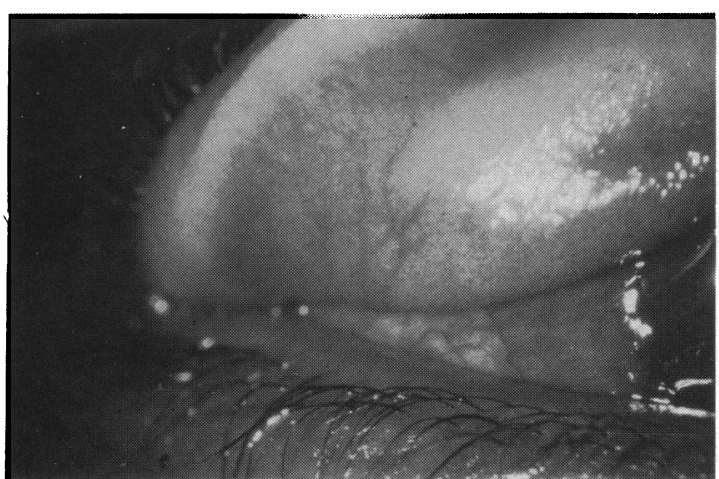

Fig. 5 13-year-old boy with active limbal $V K C$. Note the mild papillary reaction of the tarsal conjunctiva in contrast to the severe involvement of the limbus.

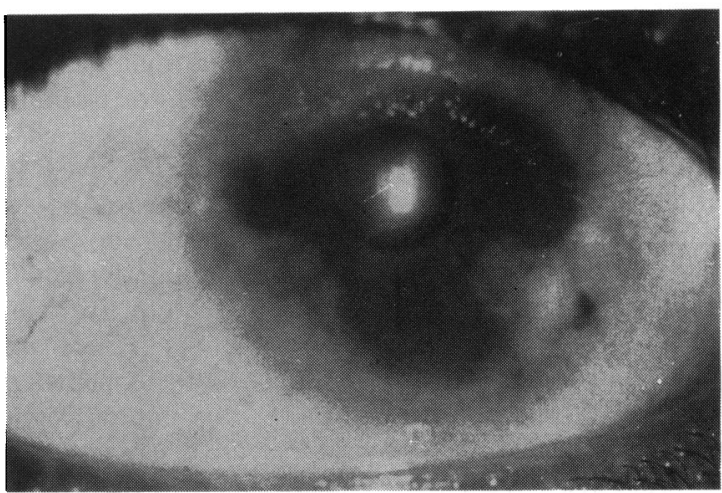

Fig. 6 16-year-old boy suffering from severe $V K C$ since age 3. The photograph was taken 2 weeks after combined treatment of SCG and dexamethasone $0.1 \%$ drops. Note the decreased pigmentation and the flat superficial scars of the pericornea.

The subjective symptoms and objective signs were totalled separately before and after treatment and the differences calculated as a percentage to show improvement or worsening of signs and symptoms.

In the placebo group (13 patients) subjective symptoms were improved by $14 \%$ only, whereas objective signs worsened by $11 \%$. These patients were switched after 4 weeks of follow-up to either dexamethasone $0.1 \% \quad(6$ patients $)$ or sodium cromoglycate ( 7 patients).

The local steroids group consisted of 20 patients of whom 14 were given initially dexamethasone $0.1 \%$ and 6 patients who were selected previously to the placebo group. Subjective symptoms were improved by $65 \%$, whereas objective signs improved by $31 \%$.

The SCG group consisted of 20 patients, of whom 13 were treated initially with SCG and 7 patients belonged previously to the placebo group. SCG improved $90 \%$ of the subjective signs and $58 \%$ of the objective signs.

An additional group of 7 patients who were severely affected by the condition and did not respond to either local steroids or SCG were treated with the combination of the 2 drugs simultaneously. They comprised 4 patients previously treated in the steroid group and 3 patients in the SCG group. In this last group response to therapy was rapid and dramatic. Within 2 weeks subjective symptoms were improved by $93 \%$ and objective signs cleared by $78 \%$. Perilimbal pigmentation decreased, and limbal lesions regressed and flattened, leaving superficial scars on peripheral cornea (Fig. 6).

\section{Discussion}

Limbal VKC is a well recognised form of the disease,${ }^{89}$ but only occasionally has it been reported 
on in detail. Burnett ${ }^{10}$ observed the condition in American Negroes in 1881 . More recently SandfordSmith" has described the condition in black patients in Nigeria, noting a range of appearance in his patients from solely a predominantly bulbar disease to solely a predominantly palpebral disease. Associated atopic conditions were not specifically investigated, but asthma or eczema were only rarely seen in these patients.

VKC has been shown to be associated with atopy. ${ }^{12}$ However, Hyams, ${ }^{13}$ Neumann, ${ }^{14}$ and Hennawi, ${ }^{3}$ dealing with patients in the Middle East, all found a low incidence of atopy and associated allergic conditions. Additionally, Hyams did not find a significant response to SCG in his patients, but this finding was not supported by Hennawi.

Of these and other patients studied in the Middle East ${ }^{4}$ some had the predominantly limbal form of the disease, but most were of the palpebral or mixed types. The purely or predominantly limbal form of VKC appears to be found more frequently in black populations. ${ }^{10} 11$ This does not appear to be related to geographic or climatic conditions, in that the entity has been described in American blacks, ${ }^{10}$ in patients in tropical Africa, " and now in temperate Africa. Moreover, in our region European patients who develop VKC appear to be susceptible to the predominantly palpebral forms of the disease, whereas Asian patients showed both limbal and palpebral lesions. Thus there would seem to be a genetic factor contributing to the form of expression of VKC in susceptible patients.

This possible genetic factor, and the low incidence of atopy found in our patients, would lead one to question whether SCG would be an effective mode of therapy in treating these patients. SCG is thought to inhibit the release of histamine and other mediators of the immediate (type I) hypersensitivity reaction in mast cells, possibly by stabilising the cell membrane to changes in $\mathrm{Ca}^{++}$ion permeability. ${ }^{1516} \mathrm{SCG}$ is not otherwise vasoactive, anti-inflammatory, or antihistaminic. It has been found to be effective in treating those diseases known to have an allergic basis. The significant response to SCG and the histopathology seen in our patients would appear to confirm the presence of such an allergic component in this form of the disease despite the absence of a positive past history of associated atopy. The inciting factors remain unknown, but exposure to ultraviolet light might be one of the causes. This factor might also explain the distribution of the pigment in the interpalpebral fissure on the exposed conjunctiva (Fig. 1) and to a less extent the male predominance, on the assumption that young boys spend more time outdoors than girls.

Hennawi showed SCG alone to be as effective as
Decadron (dexamethasone) in controlling VKC. Our study confirms this finding in previously untreated patients. Of interest is that of those patients who did not respond to either drug alone all improved markedly with the combination of SCG and steroids. Whether this finding reflects only a lack of response to one of the drugs but not to the other, or whether it represents a synergistic effect between the 2 drugs, remains to be demonstrated by further study.

Our results also confirm that the most significant response to therapy is seen symptomatically, and in the corneal limbal and bulbar pathology $y^{4-6}$ improvement on SCG occurred.

In general the improvement on SCG occurred quite rapidly, both subjectively and objectively, as was also true of steroid, contrary to some previous impressions. ${ }^{7}$ The long-term suppressant effects of SCG versus steroid in limbal VKC also deserves further study.

In conclusion, limbal VKC, as seen in black patients in Southern Africa, shares many characteristics of the classically described predominantly palpebral VKC, but there are some notable differences. The features which are comparable include: symptoms, age group, duration of the disease, male predominance, ${ }^{11}$ histopathology, ${ }^{17}$ and response to $\mathrm{SCG},{ }^{3-7}$ whereas the difference lay chiefly in the expression of the disease, which is mainly limbal.

Although in most cases the condition is benign and self-limiting, in some severely affected patients the disease is disabling and may threaten sight. These cases should be correctly diagnosed and promptly treated with relatively safe agents.

SCG eye drops are at least as effective as corticosteroids in treating the acute manifestations of the disease. The 2 together proved particularly effective in helping difficult cases that did not respond to either drug alone. It would seem reasonable, therefore, to institute treatment of severe cases or acute exacerbations with both drugs from the outset to effect a rapid response, and then to rely to SCG for long-term suppression of symptoms if necessary.

The authors thank Fisons Pharmaceuticals for supplying the Opticrom used in the trial and Dr D. Danilewitz, their medical director, for his assistance and co-operation.

\section{References}

1 Jones BR. Allergic disease of the outer eye. Trans Ophthalmol Soc UK 1971; $91: 441-7$.

2 Rice NSC, Easty D, Garner A, Jones BR, Tripathi R. Vernal keratoconjunctivitis and its management. Trans Ophthalmol Soc UK 1971; 91: 483-9.

3 Hennawi M. Clinical trial with $2 \%$ sodium cromoglycate (Opticrom) in vernal keratoconjunctivitis. Br J Ophthalmol 1980; 64: 483-6. 
4 Sayegh F, Samerra'e S, Khateeb M. Clinical trial of topical disodium cromoglycate in vernal keratoconjunctivitis. Ophthalmologia 1978; 177: 208-13.

5 Easty DL, Rice NSC, Jones BR. Disodium cromoglycate in the treatment of vernal keratoconjunctivitis. Trans Ophthalmol Soc UK 1971; 91: 491-9.

6 Tabbara KF, Arafat NT. Cromolyn effects on vernal keratoconjunctivitis in children. Arch Ophthalmol 1977; 95: 2184-6.

7 Ostler HB, Martin RG, Dawson CR. Use of disodium cromoglycate in the treatment of atopic ocular disease. Symp Ocular Ther 1977; 10: 99-108.

8 Beigelman MN. Vernal conjunctivitis. Los Angeles: University of Southern California Press. 1965.

9 Jones BR. Vernal keratitis. Trans Ophthalmol Soc UK 1961; 81: 215-28.

10 Burnett SM. Circumcorneal hypertrophy of the conjunctiva. Arch Ophthalmol 1881; 10: 414-20.
11 Sandford-Smith JH. Vernal eye disease in Northern Nigeria. Trop Geogr Med 1979; 31: 321-8.

12 Frankland AW, Easty D. Vernal keratoconjunctivitis: an atopic disease. Trans Ophthalmol Soc UK 1971; 91: 479-82.

13 Hyams SW, Bialik M. Neumann E. Clinical trial of disodium cromoglycate in vernal keratoconjunctivitis. $J$ Pediatr Ophthalmol 1975; 12: 116-8.

14 Neumann E, Gutmann MJ, Blumankrantz N, Michaelson IC. A review of four hundred cases of vernal conjunctivitis. $A m J$ Ophthalmol 1959; 47: 166-72.

15 Foreman JC, Garland LG. Cromoglycate and other anti-allergic drugs: a possible mechanism of action. Br Med J 1976; i: 820-1.

16 Spataco AC, Bosomann HB. Mechanism of action in disodium cromoglycate-mast cell calcium ion reflux after an histamine releasing stimulus. Biochem Pharmacol 1976; 25: 505-10.

17 Morgan G. The pathology of vernal conjunctivitis. Trans Ophthalmol Soc UK 1971; 91: 467-78. 